\title{
An Introduction to the Methodology of Earthquake Resistant Structures of Uniform Response
}

\author{
Mark Grigorian ${ }^{1, *}$ and Carl E. Grigorian ${ }^{2}$ \\ 1 MGA Structural Engineering Inc., 111 N. Jackson Street Glendale, CA 91206, USA \\ 2 URS Corporation, 915 Wilshire Blvd., Los Angeles, CA 90017, USA; \\ E-Mail: Carl.Grigorian@urs.com \\ * Author to whom correspondence should be addressed; E-Mail: markarjan@aol.com.
}

Received: 9 December 2011; in revised form: 22 March 2012 / Accepted: 24 April 2012 /

Published: 2 May 2012

\begin{abstract}
Structures of Uniform Response are special earthquake resistant frames in which members of similar groups such as beams, columns and braces of similar nature share the same demand-capacity ratios regardless of their location within the group. The fundamental idea behind this presentation is that seismic structural response is largely a function of design and construction, rather than analysis. Both strength and stiffness are induced rather than investigated. Failure mechanisms and stability conditions are enforced rather than tested. Structures of Uniform Response are expected to sustain relatively large inelastic displacements during major earthquakes. A simple technique has been proposed to control and address the gradual softening of such structures due to local/partial instabilities and formation of plastic hinges. In structures of uniform response, the magnitude and shape of distribution of lateral forces affects the distribution of story stiffness in proportion with story moments, therefore affecting the dynamic behavior of the system as a whole. Simple closed form formulae describing the nonlinear behavior of moment frames of uniform response have been proposed. While the scope of this contribution is limited to moment frames, the proposed method can successfully be extended to all types of recognized earthquake resisting systems.
\end{abstract}

Keywords: earthquake resistant frames; structural instability; plastic analysis; stiffness degradation; sequential hinging; energy equivalency; drift control; period analysis 


\section{Notation}

$\begin{array}{clclcl}f & \text { magnification factor } & H & \text { total building height } & T & \text { period of vibration } \\ i, j & \text { integer coordinates } & I & \text { beam moment of inertia } & U & \text { internal energy } \\ h & \text { story height } & J & \text { column moment of inertia } & V & \text { shear force } \\ \bar{h} & \text { height from base } & K & \text { sub frame stiffness } & W & \text { sub frame weight } \\ m & \text { number of stories } & L & \text { span length } & Q & \text { total weight } \\ n & \text { number of bays } & M & \text { beam moment } & \delta & \text { local displacement } \\ s & \text { order of occurrence } & N & \text { column moment } & \Delta & \text { total displacement } \\ C & \text { numerical constant } & P & \text { joint load } & \phi & \text { drift ratio } \\ E & \text { modulus of elasticity } & M^{P} & \text { beam plastic moment } & \theta & \text { joint rotation } \\ F & \text { external force } & N^{P} & \text { column plastic moment } & & \end{array}$

Indexes, superscripts and the remaining symbols are defined, as they first appear in the text.

\section{Introduction}

The purpose of this paper is to introduce the performance of Structures of Uniform Response (SUR) under lateral loading. SUR are special frameworks in which members of similar groups such as beams, columns and braces of similar physical characteristics e.g., length, end conditions etc., share the same demand-capacity ratios regardless of their location and numbers within the group. In other words, selected groups of members develop identical levels of stress and strain under similar loading conditions.

Results of inelastic static, push-over and dynamic time-history analyses [1,2], have shown that Performance Based Plastic Design methods can successfully be applied to almost all types of code recognized earthquake resisting systems. The performance of SUR as earthquake resisting systems is directly supported by these findings. In introducing SUR the paper also presents a new analytic Performance-Based Elastic-Plastic Design method for earthquake resisting moment frames, with the ability to control their response during all phases of seismic loading, starting from zero to first yield, followed by progressive plasticity up to and including incipient collapse $[3,4]$.

As far as it can be ascertained, the proposed drift increment and moment redistribution Equations are the only ones of their kind that can analytically estimate lateral displacements and element moments of such frames throughout both elastic as well as plastic ranges of loading. The step-by-step procedure presented in this work is particularly suitable for manual as well as spreadsheet computations. Most importantly, the proposed formulations help engineers gain insight into structural behavior of earthquake resistant SUR. All SUR formulations yield mathematically admissible initial designs within which member sizes can be modified for any reason, especially for meeting target objectives, optimizing material and construction costs without violating the prescribed performance conditions. The performance of SUR in the elastic and elastic-partially plastic stages satisfy all conditions of lower bound solutions and tends towards uniqueness as plasticity propagates through selected groups of members of the framework. SUR are generally more economical in the elastic range; and become lighter in total weight as the number of plastic hinges increase within the framework. Uniqueness 
implies that any upgrading of any member property can only enhance the performance of the structure beyond its targeted projections.

The methodology leading to SUR also provides a wealth of technical information that may not be readily available through traditional methods of approach. SUR in general and Moment Frames of Uniform Response (MFUR) in particular can be formulated to address the following target objectives:

- A prescribed drift ratio at any given loading or performance stage.

- A prescribed carrying capacity corresponding to any drift ratio or performance stage, including maximum allowable lateral displacement at incipient collapse.

- Predetermined sequences of formations of plastic hinges before collapse.

- Damage control in terms of the number of plastic hinges at any loading or response stage compared with number of plastic hinges at zero loading, at first yield or at incipient collapse.

- Reduction of the total weight of the structure to a theoretical minimum.

- The possibility to further enhance or the performance of the structure using moment control technologies such as brackets, haunches, end flange plates and/or proprietary devices.

\subsection{Basic Design Objectives}

The mathematical formulation of MFUR is based on the implementation of the following design objectives that:

1. The ideal inter-story drift ratio remains constant along the height of the structure, and that lateral displacements remain a linear function of the height during all phases of loading.

2. The plastic hinges are prevented from forming within columns, except at base line. Whenever possible, base line plastic hinges should form within the grade beams. Global mechanism is reached if the concept of strong-column weak-beam is considered.

3. For minimum weight MFUR, the demand-capacity ratios of all members are as close to unity as possible.

\subsection{Basic Design Assumptions}

The methodology expounded in this presentation is based on the following design assumptions:

1. Axial, shear and panel zone deformations are not coupled with flexural displacements and can be temporarily ignored for the purposes of this study.

2. Groups of similar members simultaneously resist similar types of loading or combinations of loading, e.g., flexural, axial, torsional, etc.

3. The shape of code specified distribution of earthquake forces remains constant during all loading phases. The shape could be triangular or determined by any rational analysis.

4. Initial design is based on the fundamental period of vibration of the un-degraded structure.

5. The effects of plastic hinge offsets from column center lines can be ignored.

6. The possible benefits of strain hardening and yield over-strength can be ignored.

7. Code level gravity loads have little or no effect on the ultimate carrying capacity of moment frames designed for moderate to severe earthquakes. However the columns should be designed in 
such a way as to resist gravity forces together with effects induced by plastic hinging of the beams.

8. The design earthquake loads act monotonically throughout the history of loading of the structure.

9. The frames are two dimensional and are constructed out of ductile materials and connection failure is prevented under all loading conditions.

10. The columns remain effectively elastic during all phases of loading.

Traditional design methods for earthquake resistant moment frames begin with approximate member Properties for initial sizing, and entail several cycles of analysis before a satisfactory solution is established. The proposed procedure begins with an optimized Performance-Based Elastic-Plastic design approach that already has the code prescribed criteria built into its basic algorithm. Optimization in this context implies providing as much capacity as demand imposed on or attracted by each member of the frame. Several simple examples have been provided to illustrate the applications of the proposed procedures. While the scope of the present work is limited to moment frames, the proposed method can successfully be extended to all types of recognized lateral load resisting systems.

\section{Methodology}

\section{In MFUR selected groups of beams and columns share the same drift and demand-capacity ratios.}

The most fundamental step in generating a MFUR is to select the properties of its constituent elements in such a way as to achieve geometrically similar inter story drift profiles prescribed for the entire structure during all phases of loading. In other words, the most ideal lateral deformation profile for any frame is that in which the code prescribed story level displacements fall along the same straight line i.e.,

$$
\partial_{s} \Delta_{i, s}=\partial_{s} \phi_{i, s} \cdot \bar{h}_{i}=\partial_{s} \phi_{s} \cdot \bar{h}_{i}
$$

where, $\phi_{i, s}=\phi_{s}=\Delta_{m, s} / H . \Delta_{m, s}$ is the maximum roof or $m^{\text {th }}$ level lateral displacement at $s^{\text {th }}$ response stage. Symbol $\partial_{s}$ signifies increment at $s^{\text {th }}$ consecutive iteration.

Equation (1a) indicates that points of inflexion should occur at column mid-heights. By the same token, the most ideal design drift function is that where the code prescribed inter story rotations remain the same, i.e.,

$$
\partial_{s} \phi_{i, s}=\partial_{s} \phi_{s}=\frac{\partial}{\partial \bar{h}_{i}}\left(\bar{h}_{i} / H\right) \cdot \Delta_{m, s}
$$

Theoretically there can be as many loading or response stages as there are beams, i.e., $s_{\max .}=m \times n$. However, $s_{\text {max. }}=n$ or $s_{\text {max. }}=m$ offer more practical options for design purposes. Rotation $\phi_{m, s=1}=\phi_{Y}$ may be construed as the initial target drift corresponding to initial target displacement $\Delta_{m, s=1}=\Delta_{Y}$ at first yield. The line diagram of a regular MFUR together with its idealized design displacement profiles, subjected to a generalized distribution of lateral forces, is presented in Figure 1. The design conditions (1a) and (1b) imply equal incremental joint rotations for all members of the frame i.e.,

$$
\partial_{s} \theta_{G . i, s}=\partial_{s} \theta_{G . s}
$$


Figure 1. Laterally Loaded Moment Frames of Uniform Response (MFUR) with Linearly Varying Drift Profiles.

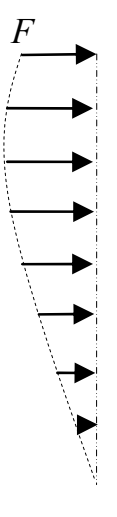

(1a)

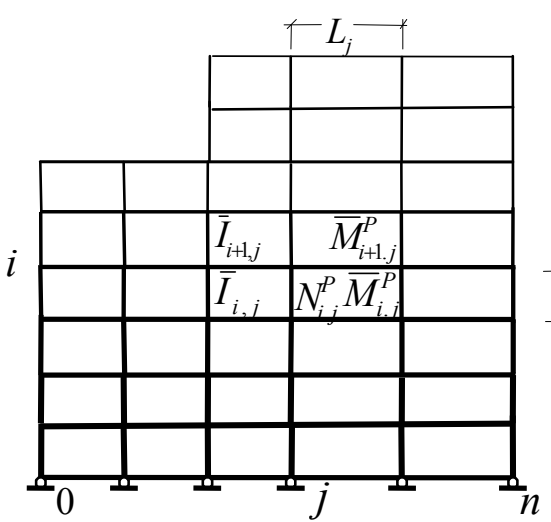

(1b)

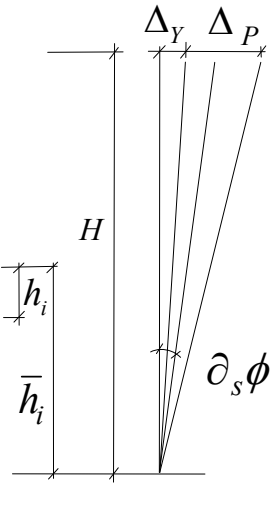

(1c)

The global equilibrium of the structure in terms of beam stiffness and global frame rotation $\theta_{G, s}$ at any stage " $s$ " can be expressed as:

$$
\sum_{i=1}^{m} \partial_{s} V_{i, s} h_{i}=\sum_{i=1}^{m} \partial_{s} F_{i, s} \bar{h}_{i}=\sum_{i=0}^{m} \sum_{j=1}^{n} 2 \partial_{s} M_{B . i, j, s}=12 E \partial_{s} \theta_{G, s} \sum_{i=0}^{m} \sum_{j=1}^{n} k_{i, j}
$$

Where, $V_{i, s}, k_{i, j}=(I / L)_{i, j}, M_{B . i, j, s}$ and $I_{i, s}$ are the story level shear force, relative stiffness, end moments and moment of inertia of beam "i,j” respectively. Equation (2a) directly yields the global rotation of the structure as;

$$
\partial_{s} \theta_{G . s}=\sum_{i=1}^{m} \partial_{s} V_{i, s} h_{i} / 12 E \sum_{i=0}^{m} \sum_{j=1}^{n} k_{i, j}
$$

Similarly, the racking equilibrium Equation of any representative floor in terms of its beam stifnesses may be expressed as:

$$
\partial_{s} M_{i, s}^{A}=\sum_{j=1}^{n} 2 \partial_{s} M_{B . i, j, s}=12 E \partial_{s} \theta_{B, s} \sum_{j=1}^{n} k_{i, j}
$$

The quantities $M_{i, s}^{A}=\left(M_{i, s}^{R}+M_{i+1, s}^{R}\right) / 2=\left(V_{i, s} h_{i}+V_{i+1, s} h_{i+1}\right) / 2$ and $2 M_{i, s}^{A}$ are defined as the average and total racking moment acting on $i^{\text {th }}$ level beams at $s^{\text {th }}$ response stage respectively. $M_{1, s}^{A}=M_{1, s}^{R}=V_{1, s} h_{1} / 2$, and $M_{m, s}^{A}=M_{m, s}^{R}=V_{m, s} h_{m} / 2$ correspond to average racking moments of grade and roof level beams respectively. $M_{i, s}^{R}=V_{i, s} h_{i}$ is defined as the $i^{\text {th }}$ story raking moment at $s^{\text {th }}$ response stage. Equation $(2 \mathrm{c})$ in turn directly yields the floor level rotations as:

$$
\partial_{s} \theta_{B, s}=\partial_{s} M_{i, s}^{A} / 12 E \sum_{j=1}^{n} k_{i, j}
$$

However, since $\partial_{s} \theta_{G . i, s}=\partial_{s} \theta_{B . i, s}$, then equating the global rotation Equation (2b), and floor level rotation, Equation (2d), gives:

$$
\partial_{s} M_{i, s}^{A} / \sum_{j=1}^{n} k_{i, j}=\sum_{i=1}^{m} \partial_{s} V_{i, s} h_{i} / \sum_{i=0}^{m} \sum_{j=1}^{n} k_{i, j}
$$


Substituting for $\sum_{i=1}^{m} \partial_{s} V_{i, s} h_{i}=\sum_{i=0}^{m} \partial_{s} M_{i, s}^{A}$ and expanding the right hand side of Equation (3a), it gives:

$$
\frac{\partial_{s} M_{i, s}^{A}}{\sum_{j=1}^{n} k_{i, j}}=\frac{\partial_{s} M_{1, s}^{A}+\partial_{s} M_{2, s}^{A}+\ldots . \partial_{s} M_{i, s}^{A}+\ldots . \partial_{s} M_{m, s}^{A}}{\sum_{j=1}^{n} k_{1, j}+\sum_{j=1}^{n} k_{2, j}+\ldots \ldots . \sum_{j=1}^{n} k_{i, j} \ldots \ldots . .+\sum_{j=1}^{n} k_{m, j}}
$$

Equation (3b) can be satisfied only if the following mathematical conditions are met:

$$
\partial_{s} M_{i, s}^{A} / \sum_{j=1}^{n} k_{i, j}=\partial_{s} M_{1, s}^{A} / \sum_{j=1}^{n} k_{1, j} \ldots \ldots=\partial_{s} M 2_{i, s}^{A} / \sum_{j=1}^{n} k_{2, j} \ldots=\partial_{s} M_{m, s}^{A} / \sum_{j=1}^{n} k_{m, j}
$$

In physical terms, the condition of uniform drift requires that the sum of the stiffnesses of beams of each floor be selected in proportion with the average racking moments of that floor, i.e.,

$$
\sum_{j=1}^{n} k_{1, j}=\frac{\partial_{s} M_{1, s}^{A}}{\partial_{s} M_{m, s}^{A}} \sum_{j=1}^{n} k_{m, j} \ldots \sum_{j=1}^{n} k_{i, j}=\frac{\partial_{s} M_{i, s}^{A}}{\partial_{s} M_{m, s}^{A}} \sum_{j=1}^{n} k_{m, j}
$$

Equation (3d) also describes a state of uniform demand-capacity for the beams of the structure. In practical terms, uniform demand-capacity implies providing as much capacity as demand imposed on or attracted by each member of the frame. Since equal joint rotations also imply zero moments at column mid-points, then the racking equilibrium of the frame in terms of column moments of any representative floor " $m$ " or " $i$ " may be expressed as;

$$
\partial_{s} V_{m, s} h_{m}=\partial_{s} M_{m, s}^{R}=\sum_{j=0}^{n} 2 \partial_{s} M_{C . m, j, s}=12 E \partial_{s} \theta_{C . s} \sum_{j=0}^{n} \bar{k}_{m, j}
$$

or

$$
\partial_{s} V_{i, s} h_{i}=\partial_{s} M_{i, s}^{R}=\sum_{j=0}^{n} 2 \partial_{s} M_{C . i, j, s}=12 E \partial_{s} \theta_{C . s} \sum_{j=0}^{n} \bar{k}_{i, j}
$$

Where, $\bar{k}_{i, j}=(J / h)_{i, j}, M_{C . i, j, s}$, and $J_{i, j}$ are the relative stiffness, end moments and moment of inertia of column " $i, j$ ” respectively. Equation (4b) yields the floor level rotations as:

$$
\partial_{s} \theta_{C, s}=\partial_{s} M_{i, s}^{R} / 12 E \sum_{j=0}^{n} \bar{k}_{i, j}
$$

$\theta_{C . s}$ is the drift component of the $i^{\text {th }}$ level floor, due to deformations of the columns of the same level. Comparing the two sides of Equations (4a) and (4c) yields the conditions of uniform response or demand-capacity for the columns of the subject frame as:

$$
\sum_{j=0}^{n} \bar{k}_{1, j}=\frac{\partial_{s} M_{1, s}^{R}}{\partial_{s} M_{m, s}^{R}} \sum_{j=1}^{n} \bar{k}_{m, j} \ldots \ldots \sum_{j=0}^{n} \bar{k}_{i, j}=\frac{\partial_{s} M_{i, s}^{R}}{\partial_{s} M_{m, s}^{R}} \sum_{j=1}^{n} \bar{k}_{m, j}
$$

Equations (3d) and (4d) together fulfill the condition of compatible drift angles along the height of the frame and verify the statement of the methodology of MFUR presented at the beginning of this section. The applications of the proposed approach are elaborated through parametric examples in the forthcoming sections. 


\section{Story Level Elastic-Plastic Displacement Response}

The total drift of any story in terms of its racking moments at any response stage may be computed as;

$$
\partial_{s} \phi_{i, s}=\partial_{s} \theta_{C . i, s}+\partial_{s} \theta_{B . i, s}
$$

or

$$
\partial_{s} \phi_{i, s}=\partial_{s} M_{i, s}^{R} / 12 E \sum_{j=0}^{n} \bar{k}_{i, j}+\partial_{s} M_{i, s}^{A} / 12 E \sum_{j=1}^{n} k_{i, j}
$$

In the absence of gravity loads, the force-deformation relationship or the drift increment Equation (5b) may be expanded to include the effects of member plasticity and story level axial forces, [5,6] i.e.,

$$
\partial_{s} \phi_{i, s}=\frac{\partial_{s} V_{i, s} h_{i}}{12 E f_{C R . i, s}}\left[\frac{1}{\sum_{j=0}^{n} \bar{k}_{i, j}}+\frac{1}{\sum_{j=1}^{n} k_{i, j}}\right]+\frac{\partial_{s} V_{i+1, s} h_{i+1}}{12 E f_{C R . i, s}}\left[\frac{1}{\sum_{j=1}^{n} k_{i, j}}\right]
$$

However, since drift increment is a function of stiffness degradation as well as sequence of formation of plastic hinges, it becomes necessary to relate beam stiffness factors $k_{i, j}$ to their sequence of formation of plastic hinges or response stage " $s$ ", by means of subscript " $r$ ", rather than their location " $j$ ". This is achieved by replacing $k_{i, j}$ with $k_{i, s}$ and $\sum_{j=1}^{n} k_{i, j}$ with $\sum_{r=1}^{n} k_{i, r}$ and incorporating the symbol $\delta_{r}^{s-1}$ and $\bar{\delta}_{j}$ in Equation (5c) in order to include the effects of formation or prevention of formation of plastic hinges at the ends of beams " $i, s$ ".i.e.,

$$
\partial_{s} \phi_{s, i}=\frac{\partial_{s} V_{i, s} h_{i}}{12 E f_{C R . i, s}}\left[\frac{1}{\sum_{j=0}^{n} \bar{\delta}_{j} \bar{k}_{i, j}}+\frac{1}{\sum_{r=1}^{n} \delta_{r}^{s-1} k_{i, r}}\right]+\frac{\partial_{s} V_{i+1, s} h_{i+1}}{12 E f_{C R . i, s}}\left[\frac{1}{\sum_{r=1}^{n} \delta_{r}^{s-1} k_{i, r}}\right]
$$

Equation (5d) now represents both the elastic as well as plastic deformations of the subject moment frame within a single, seamless expression, where by definition, the smaller the subscript " $s$ " the stiffer the beam it represents. $\delta_{r}^{s-1}=0$ for $M_{B . i, s}=M_{B . i, s}^{P}$ and implies structural damage and/or loss of stiffness with respect to beam " $i, s^{\prime} . \delta_{r}^{s-1}=1$ for $M_{B . i, s}<M_{B . i, s}^{P}$. In mathematical terms, $\delta_{r}^{s-1}=1$ for $r>s-1$, and $\delta_{r}^{s-1}=0$ for $r \leq s-1$. Similarly the symbol $\bar{\delta}_{j}$ has been introduced to relate column stifnesses $\bar{k}_{i, j}$ to effects of formation of plastic hinges in the adjoining beams " $i, j$ " and " $i, j-1 . \bar{\delta}_{j}=0$ for $M_{B . i, j}=M_{B . i, j}^{P}$ and $M_{B . i, j-1}=M_{B . i, j-1}^{P}$, otherwise $\bar{\delta}_{j}=1 . f_{C R . i, s}=\left[1-\left(\sum_{j=0}^{n} P_{i, j}\right) / P_{C R . i, s}\right]$ is the force magnification function. $\sum_{j=0}^{n} P_{i, j}$ and $P_{C R i, s}=K_{i, s} h_{i}$ are the total axial load and the critical axial load of level " $i$ " at $s^{\text {th }}$ response stage respectively. In reality, since the drift ratio is constant, it would be sufficient to compute $\partial_{s} \phi_{s, i}=\partial_{s} \phi_{s}$ for the simplest representative level, i.e., the roof, where, $V_{m+1, s}=h_{m+1}=0$. Equation $(5 \mathrm{~d})$ reduces to: 


$$
\partial_{s} \phi_{m, s}=\partial_{s} \phi_{s}=\frac{\partial_{s} V_{m, s} h_{m}}{12 E f_{C R, m, s}}\left[\frac{1}{\sum_{j=0}^{n} \bar{\delta}_{j} \bar{k}_{m, j}}+\frac{1}{\sum_{r=1}^{n} \delta_{r}^{s-1} k_{m, r}}\right]=\frac{\partial_{s} V_{m, s}}{K_{m, s} h_{m}}
$$

$K_{m, s}$ is the stiffness of the $m^{\text {th }}$ level framework at $s^{\text {th }}$ response stage.

\subsection{Demonstrative Example I}

Consider the lateral displacements of the four bay $(n=4)$, three story $(m=3)$ moment frame of Figure (2a), subjected to a uniform distribution of lateral forces $F_{i}=F$ and axial joint forces $P_{i, 0}=P_{i, 4}=P / 2$ for all " $i$ " and $P_{i, j}=P$ for all other " $i, j$ ".

Figure 2. Example I, Moment Frame Loading and Racking Moment.

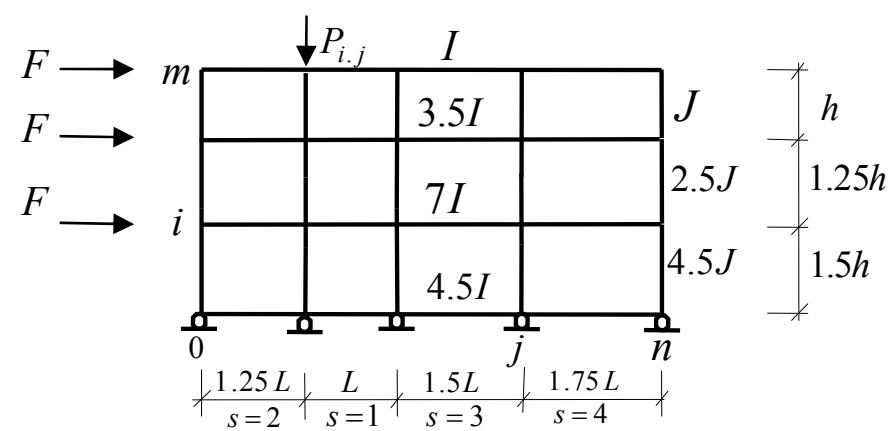

(2a)

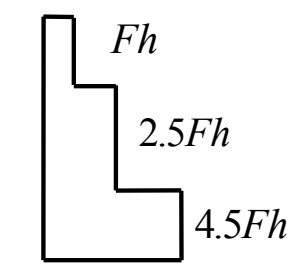

$M_{O}=\sum V h=8 F h$

The cross section and moment of resistance of beams and columns of each level are required to be constant, e.g., $I_{3, j}=I$ and $M_{3, j}^{P}=M^{P}$ for all " $j$ ". $J_{3,0}=J_{3,4}=J$ and $J_{i, j}=2 J_{i}$ for all other " $j$ ". The primary purpose of this exercise is to generate a MFUR by computing the quantities $I_{i}, J_{i}$ and $M_{i}^{P}$ in terms of their corresponding values $I_{3}$, and $M_{3}^{P}$ (at roof level) respectively. The distribution of story level racking moments $M_{i, s}^{R}$ is shown in Figure (2b). The total racking moments $2 M_{i, s}^{A}$ can now be computed as $4.5 F_{s} h, 7.0 F_{s} h, 3.5 F_{s} h$ and $1.0 F_{s} h$ for the grade, $1^{\text {st }}, 2^{\text {nd }}$ and roof level beams respectively. Since $I_{i}$ is uniform for each level and the quantity $\sum_{r=1}^{4}(1 / L)_{i, r}=3.0381 / L$ is constant for the entire frame, then for $s=1 ; \sum_{j=0}^{n} \bar{\delta}_{j}(1 / h)_{i, j}=8 J_{i} / h$ and $\sum_{r=1}^{n} \delta_{r}^{s-1} k_{i, r}=I_{i} \sum_{r=1}^{4}(1 / L)_{i, r}=3.0381 I_{i} / L$. And, as a result, Equation (3d) reduces to $I_{i}=\left(M_{i, s=1}^{A} / M_{3, s=1}^{A}\right) I_{3}$, i.e., $I_{0}=\left(4.5 F_{1} h / F_{1} h\right) I=4.5 I$, $I_{1}=\left[\left(4.5 F_{1} h+2.5 F_{1} h\right) / F_{1} h\right] I=7 I, I_{2}=\left[\left(2.5 F_{1} h+1.0 F_{1} h\right) / F_{1} h\right] I=3.5 I$, and $I_{3}=I$. By the same token, since $\sum_{j=0}^{n} \bar{k}_{i=3, j}=2 n J / h \quad$ simplifies Equation (4d) to $J_{i}=\left(M_{i, s=1}^{R} / M_{m, s=1}^{R}\right) J_{m}$ then: $J_{1}=\left(4.5 F_{1} h / F_{1} h\right) J=4.5 J, J_{2}=\left(2.5 F_{1} h / F_{1} h\right) J=2.5 J J_{3}=J$. The third level (roof) drift of the newly generated MFUR at $s=1$ can now be expressed as:

$$
\phi_{3, s=1}=\phi_{s=1}=\frac{V_{3, s=1} h}{12 E f_{C R .3, s=1}}\left[\frac{h}{8 J}+\frac{L}{3.0381 I}\right]=\frac{V_{3, s=1}}{K_{3, s=1} h}
$$


Assuming that the design decisions; $N_{i, 1}^{P}=N_{i, 4}^{P}>M_{i, 1}^{P}, N_{i, j}^{P}>2 M_{i, j}^{P}$ for all other " $j$ " an $J_{i}=1.2 I_{i} \mathrm{~d}$ satisfy the Strong column-weak beam requirements, then for $f_{C R .3, j}=0.80, L=h, \delta_{r}^{-1=0}=1$ and $V_{3, s=1}=F_{1}$, Equation (6a) becomes;

$$
\phi_{s=1}=\frac{F_{1} h^{2}}{12 \times 0.8 E I}\left[\frac{1}{9.6}+\frac{1}{3.0381}\right]=\frac{0.0451 F_{1} h^{2}}{E I}=\frac{F_{1}}{K_{s=1} h}
$$

Therefore; $\Delta_{i=1, s=1}=\phi_{s=1} 1.5 h=0.0677 F_{s=1} h^{3} / E I, \Delta_{i=2, s=1}=\phi_{s=1} 2.75 h=0.1241 F_{s=1} h^{3} / E I$ and

$\Delta_{i=3, s=1}=\phi_{s=1} 3.75 h=0.1693 F_{s=1} h^{3} / E I$. Finally, if the target drift $\phi_{s=1}$ is not to exceed $\phi_{Y}$, where the subscript $Y$ signifies first yield, i.e., $F_{1}=F_{Y}$ then, the design representative moment of inertia becomes $I=I_{Y}=0.0451 F_{Y} h^{2} / \phi_{Y} E$.

\section{Story Level Elastic-Plastic Moment Response}

The elastic-plastic displacement response of the moment frame, Equation (5c), is directly influenced by the redistribution of forces in the members of the structure. For instance, if the magnitude of the end moments of beam " $i, j$ " at $s$ th response stage is given by; $\partial_{s} M_{B . i, s}=6 E k_{i, s} \partial_{s} \theta_{B . s}$, then by substituting for $\partial_{S} \theta_{B . S}$ from Equation (2d) gives:

$$
\partial_{s} M_{B . i, s}=\frac{\partial_{s} M_{i, s}^{A} \times k_{i, s}}{2 f_{C R . i, s} \sum_{r=1}^{n} \delta_{r}^{s-1} k_{i, r}}
$$

and

$$
\partial_{r} N_{C . i, j, s}=\partial_{s} M_{B . i, j-1, s}+\partial_{s} M_{B . i, j, s}
$$

as the Moment Redistribution or Plasticity Progression equations of the beams and columns of the subject frame at any given response stage respectively. At ultimate loading or incipient collapse the quantities $\phi_{i, s}, F_{i, s}, V_{i, s}, M_{i, s}^{A}$ and $M_{B . i, s}$ become $\phi_{i}^{P}, F_{i}^{P}, V_{i}^{P}, M_{i}^{A P}$ and $M_{i, s}^{P}$ respectively.

The ultimate carrying capacity of regular moment frames is usually computed using the virtual work method of plastic analysis, [7] which eventually results in static equilibrium Equations that involve the global overturning moments $M_{O}$ of the system at incipient collapse, e.g., considering the plastic collapse of the moment frame of Figure (3d), through formation of plastic hinges at beam ends only, and conforming to a uniform virtual side sway of inclination $\theta=1$, it gives:

$$
\sum_{i=0}^{m} M_{i}^{R P}=\sum_{i=1}^{m} V_{i}^{P} h_{i}=\sum_{i=1}^{m} F_{i}^{P} \bar{h}_{i}=M_{O}^{P}=\sum_{i=0}^{m} \sum_{j=1}^{n} 2 M_{i, j}^{P}
$$

For the particular conditions of Example I, the long hand solution of Equation (8a) gives; $[3.75+2.75+1.5] F^{P} h=2[1.0+3.5+7.0+4.5] n M^{P}$ or $F^{P}=4 n M^{P} / h$ as the ultimate load carrying capacity of the subject moment frame. However in case of MFUR, the racking equilibrium Equation of any story, Equation (2c), can also be used to achieve the same results, i.e.,

$$
M_{i}^{A P}=\sum_{j=1}^{n} 2 M_{i, j}^{P}=2 M_{i}^{P}\left(u_{i, 1}+\ldots+1+\ldots u_{i, j}+\ldots u_{i, m}\right)
$$




$$
M_{m}^{A P}=\sum_{j=1}^{n} 2 M_{m, j}^{P}=2 M_{m}^{P}\left(u_{i, 1}+\ldots+1+\ldots u_{i, j}+\ldots u_{i, m}\right)
$$

Where, $M_{i}^{P}$ is the plastic moment of resistance of the stiffest beam of the $i^{\text {th }}$ level framing. Since the pre-assigned uniformity ratios $u_{0, j}=\ldots=u_{i, j} \ldots=u_{m, j}$ are constant for all " $i$ ”, then dividing Equations (8a) and ( $8 b)$ by each other, reaffirms the condition of uniform strength at incipient collapse, i.e.,

$$
M_{i}^{P}=\left(M_{i}^{A P} / M_{m}^{A P}\right) M_{m}^{P}
$$

Figure 3. Progression of Plasticity in Moment Frame of Uniform Response.

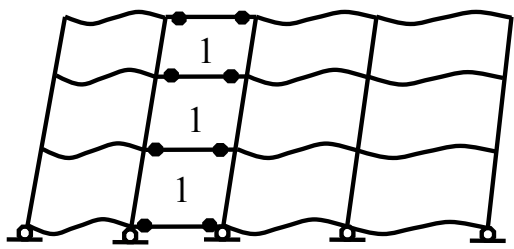

(3a) Stage -1

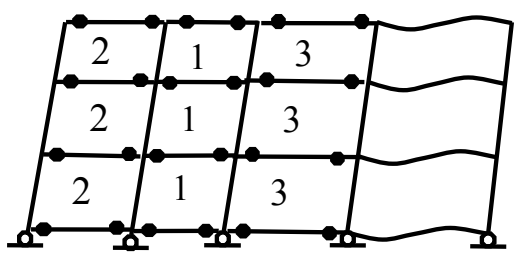

(3a) Stage -3

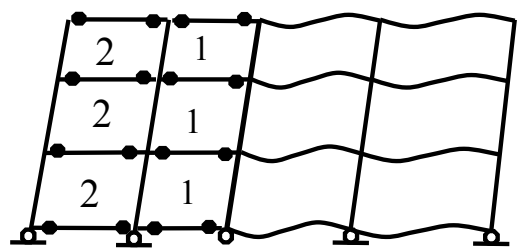

(3a) Stage -2

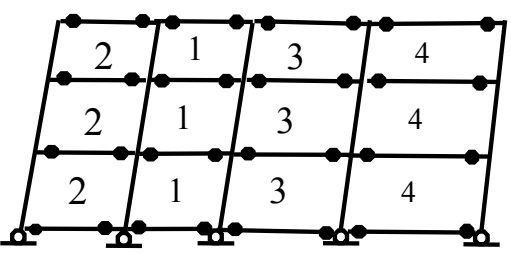

(3a) Stage -4

Equation ( $8 \mathrm{~d}$ ) can be used to compute the plastic moments of resistance of beams of any level " $i$ " in terms of plastic moments of resistance of the uppermost level beams. For the MFUR of the preceding example this gives; $M_{0}^{P}=4.5 M^{P}, \quad M_{1}^{P}=7 M^{P}, \quad M_{2}^{P}=3.5 M^{P}$ and $M_{3}^{P}=M^{P}$. Substituting $M_{m}^{A P}=F^{P} h / 2$ and $\sum_{j=1}^{n}\left(u_{i, j}=1\right)=n$ in Equation (8c) yields; $F^{P} h / 2=2 n M^{P}$ or $F^{P}=4 n M^{P} / h$, a result already established using Equation (8a) above. This result implies that:

The ultimate load carrying capacity of an $(m \times n)$ MFUR with moment resisting grade beams under lateral loading of apex value $F^{P}$ is $M^{P}=F^{P} h / 4 n$.

In physical terms, the plastic failure load of MFUR with moment resisting grade beams is independent of the number of stories and the distribution profile of the lateral forces. Equation (7a) can now be used to establish the first, $r=1$, increment of loading that causes formation of the first set of plastic hinges in the beams of the stiffest bay of the structure. Since the plastic hinges of the beams of any bay " $j$ " form simultaneously, it would suffice to first study the distribution of moments of any level " $i$ " and then extend the results to beams of other bays by simple proportioning as indicated by Equation (8d), i.e.,

$$
\partial_{1} M_{B . m, s=1}=\frac{\partial_{1} M_{m, 1}^{A} k_{m, s=1}}{2 f_{C R . m, 1} \sum_{r=1}^{n} \delta_{r}^{s-1} k_{m, r}}
$$


Substituting for $\partial_{1} M_{m, s=1}^{A}=\partial_{1} V_{m, s=1} h_{m} / 2=\partial_{1} F_{m, s=1} h_{m}=\partial_{1} F_{m, 1} h, \partial_{1} M_{B . m, s=1}=M^{P}$ and $\delta_{r}^{s=1}=\delta_{r}^{1-1=0}=1$ in Equation (9a) after some rearrangement, it gives the amount of force needed to produce the first set of plastic hinges in the stiffest beam of the $m^{\text {th }}$ level:

$$
\partial_{1} F_{m, 1}=\frac{4 M^{p}}{k_{m, 1} h_{m}} f_{C R . m, 1} \sum_{r=1}^{n} k_{m, r}
$$

Now bearing in mind that by virtue of Equation (7a) moments generated in the $x^{\text {th }}$ beam $(x>s)$ of any level can be expressed in terms of the maximum moments of the stiffest beam of that level i.e., $\partial_{s} M_{B . m, s=x}=\left(k_{m, s=x} / k_{m, s}\right) M_{i}^{P}$ and that the sequence of formation of the plastic hinges of any level is the same as the sequence of decreasing values of stiffnesses of the beams of the same floor, then the plastic moment of resistance of the stiffest element $s=1$ and moment of resistance of the next stiffest element $s=2$ can be computed as $M^{P}$ and $\left(k_{m, s=2} / k_{m, s=1}\right) M^{p}<M^{P}$ respectively. Therefore, the balance of bending moment needed to elevate the moment of resistance of beam $s=2$ to $M^{p}$ can be computed as $\left[1-\left(k_{m, s=2} / k_{m, s=1}\right)\right] M^{p}$, whence the amount of additional force required to generate plastic hinges at the ends of the next stiffest beam may be generalized as:

$$
\partial_{s} F_{m, s}=\frac{4 M^{p}}{k_{m, s} h_{m}} f_{C R . m, s}\left(1-\frac{k_{m, s}}{k_{m, s-1}}\right) \sum_{r=1}^{n} \delta_{r}^{s-1} k_{m, r}
$$

Since the sum of the incremental forces $\partial_{s} F_{m, s}$ should add up to the ultimate load $F_{m}^{P}=F^{P}$, then summing both sides of Equation (9c) over all " $n$ " iterations gives:

$$
\sum_{s=1}^{n} \partial_{s} F_{m, s}=\sum_{s=1}^{n} \frac{4 M^{P}}{k_{m, s} h_{m}} f_{C R . m, s}\left(1-\frac{k_{m, s}}{k_{m, s-1}}\right) \sum_{r=1}^{n} \delta_{r}^{s-1} k_{m, r}
$$

Substituting for $\sum_{s=1}^{n}\left(\frac{1}{k_{m, s}}-\frac{1}{k_{m, s-1}}\right) \sum_{r=1}^{n} \delta_{r}^{s-1} k_{m, r}=n, \quad \sum_{s=1}^{n} \partial_{s} F_{m, s}=F_{m}^{P}=F^{P}$ and $f_{C R . m, s}=1$ in Equation (9d) leads to the previously established solution; $F^{P}=4 n M^{P} / h$.

Equations (5e) and (9c) indicate that each stage of propagation of plastic hinges characterized by $s=1,2 \ldots, n$ may be construed as a target design point or a state of stable damage with respect to fully elastic or fully plastic conditions of the structure. The final stage also represents a minimum weight, unique [8] state of plastic design since it satisfies the prescribed yield criteria, and static equilibrium as well as the selected boundary support conditions at incipient collapse. This implies that the proposed scheme also provides an envelope of several initial designs within which member sizes could be rearranged for any purpose while observing the prescribed performance conditions.

\subsection{Demonstrative Example II}

Use Equations (9c) and (5e) to study the nonlinear behavior of the MFUR of the previous example and compute the total internal energy of the system in terms of the drift function $\phi$. Given; $k_{3, s=1}=I / L, \quad k_{3, s=2}=0.8 I / L, \quad k_{3, s=3}=0.6667 I / L, \quad k_{3, s=4}=0.5714 I / L, J=1.2 I, s_{\text {max. }}=n=4$, $V_{3, s}=F_{3, s}, h=L$ and $P_{i, j}=0$. Hence from Equation (9c): 


$$
\partial_{s} F_{3, s}=\frac{4 M^{p}}{k_{3, s} h_{3}}\left(1-\frac{k_{3, s}}{k_{3, s-1}}\right) \sum_{r=1}^{n=4} \delta_{r}^{s-1} k_{3, r}
$$

In other words:

$$
\begin{aligned}
& \partial_{1} F_{3,1}=\left(4 M^{P} / h\right) 1.00 \times(1-0.0000)[1.0+0.8+0.6667+0.5714]=12.1524 M^{P} / h . \\
& \partial_{2} F_{3,2}=\left(4 M^{P} / h\right) 1.25 \times(1-0.8000)[0.0+0.8+0.6667+0.5714]=2.0381 M^{P} / h . \\
& \partial_{3} F_{3,3}=\left(4 M^{P} / h\right) 1.50 \times(1-0.8334)[0.0+0.0+0.6667+0.5714]=1.2376 M^{P} / h . \\
& \partial_{4} F_{3,4}=\left(4 M^{P} / h\right) 1.75 \times(1-0.8571)[0.0+0.0+0.0000+0.5714]=0.5717 M^{P} / h .
\end{aligned}
$$

Therefore, $\quad F_{1}=12.1524 M^{p} / h, \quad F_{2}=14.1905 M^{p} / h, \quad F_{3}=15.4281 M^{p} / h \quad, \quad$ and as expected $F_{4}=\sum_{s=1}^{n} \partial_{s} F_{3, s}=F^{P}=16 M^{P} / h$, reconfirms the validity of the failure load formula $F^{P}=4 n M^{P} / h$.

It is instructive to note that because of the particular sequence of formation of plastic hinges, all columns remain intact up to and including completion of stage two, i.e., $\bar{\delta}_{j}=1$ for $s=1$ and $s=2$. After culmination of stage two, first columns $j=0$ and $j=1$ together at the beginning of $s=3$, next columns $j=2$ at the end of $s=3$, then columns $j=3$ and $j=4$ after culmination of $s=4$ lose their stiffness, due to formation of plastic hinges at their adjoining beam ends. Equation (5e) for drift increment becomes:

$$
\partial_{s} \phi_{3, s}=\frac{F_{3, s} h}{12 E}\left[\frac{1}{\sum_{j=0}^{n} \bar{\delta}_{j}(9.6 I / L)}+\frac{1}{\sum_{r=1}^{n=4} \delta_{r}^{s-1} k_{m, s}}\right]
$$

Or in numerical terms:

$$
\begin{aligned}
& \partial_{1} \phi_{3,1}=\frac{F_{3,1} h L}{12 E I}\left[\frac{1}{9.6}+\frac{1}{3.0381}\right]=\frac{12.1524 \times 0.0361 M^{P} L}{E I}=0.4388 \frac{M^{P} L}{E I} \\
& \partial_{2} \phi_{3,2}=\frac{F_{3,2} h L}{12 E I}\left[\frac{1}{9.6}+\frac{1}{2.0381}\right]=\frac{2.0381 \times 0.0496 M^{P} L}{E I}=0.1010 \frac{M^{P} L}{E I} \\
& \partial_{3} \phi_{3,3}=\frac{F_{3,3} h L}{12 E I}\left[\frac{1}{6.0}+\frac{1}{1.2381}\right]=\frac{1.2376 \times 0.0812 M^{P} L}{E I}=0.1005 \frac{M^{P} L}{E I} \\
& \partial_{4} \phi_{3,4}=\frac{F_{3,4} h L}{12 E I}\left[\frac{1}{4.8}+\frac{1}{0.5714}\right]=\frac{0.5714 \times 0.1632 M^{P} L}{E I}=0.0933 \frac{M^{P} L}{E I}
\end{aligned}
$$

Therefore; $\quad \phi_{1}=\phi_{Y}=0.4388\left(M^{P} L / E I\right), \quad \phi_{2}=0.5398\left(M^{P} L / E I\right), \quad \phi_{3}=0.6403\left(M^{P} L / E I\right) \quad$ and $\phi_{4}=\phi^{P}=0.7336\left(M^{P} L / E I\right)$. The combined numerical results of groups of Equations (10b) and (11b), are presented in Figure (4) as the nonlinear load-displacement relationship of the subject MFUR. Equations (5c) and (9e) together provide useful design information that neither elastic nor plastic methods of analysis can offer on their own, for instance the maximum lateral displacement of the example frame at first yield and incipient collapse can be estimated as:

$$
\Delta_{Y .}=\Delta_{3,1}=0.4388(F h L / 16 E I) \times 3.75 h=F h^{2} L / 9.72 E I
$$


and

$$
\Delta_{P}=\Delta_{3,4}=0.7336(F h L / 16 E I) \times 3.75 h=F h^{2} L / 5.82 E I
$$

respectively. Furthermore, it was demonstrated that the sequences of formations of plastic hinges could be controlled by selecting the relative stiffness of groups of similar beams in accordance with certain target decisions.

Figure 4. Load-Displacement relationship, Demonstrative Example II.

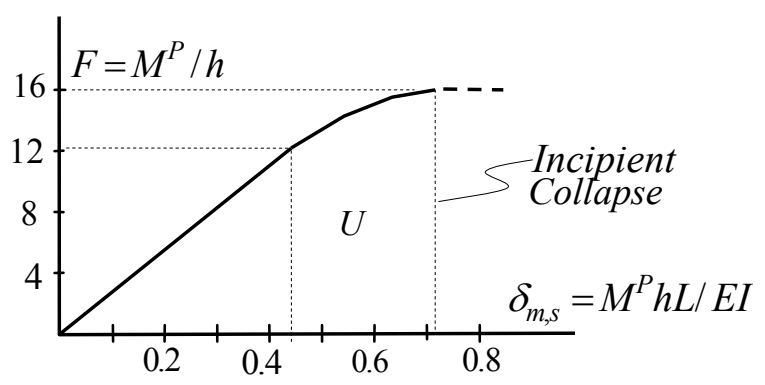

\section{Energy Computations For MFUR}

The total accumulative internal energy of any stable structural system due to elastic-plastic deformations of its constituent elements at any response stage can be computed as:

$$
U_{s}=\sum_{i=1}^{m} \sum_{r=1}^{s}\left(F_{i, r-1}+F_{i, r}\right) \partial_{r} \phi_{i, r} / 2
$$

However, since in MFUR the drift ratio $\phi_{s}$ is constant, it would suffice to compute the internal energy of any representative level, such as that of the $m^{\text {th }}$ level, and then compute the rest by simple proportioning. The energy Equation corresponding to level " $m$ " may be computed as:

$$
U_{m, s}=\sum_{r=1}^{s} U_{m, r}=\sum_{r=1}^{s}\left(F_{m, r-1}+F_{m, r}\right) \partial_{r} \phi_{m, r} / 2
$$

Furthermore, since the ratio of internal energies of any two stories is the same as the ratio of average racking moments of the same two stories, i.e., $U_{i, s}=\left(M_{i, s}^{A} / M_{m, s}^{A}\right) U_{m, s}$, then the total energy of the system may be expressed as:

$$
U_{s}=\sum_{i=1}^{m}\left(U_{m, s} / M_{m, s}^{A}\right) M_{i, s}^{A}=\left(U_{m, s} / M_{m, s}^{A}\right) \sum_{i=1}^{n} M_{i, s}^{R}
$$

Recalling that the sum of the average racking moments is equal to the sum of the story racking moments as well as the global overturning moment, i.e., $\sum_{i=1}^{m} M_{i, s}^{A}=\sum_{i=1}^{n} M_{i, s}^{R}=M_{O, s}$, then Equation (12c) reduces to its most practical form:

$$
U_{s}=\left(U_{m, s} / M_{m, a}^{R}\right) M_{O, s}
$$

Equation (12d) implies that: 
The ratio of total internal energy of MFUR to that of anyone of its levels, such as the roof, is equal to the ratio of the global overturning moment to the overturning moment of that (roof) Level.

A proof of the validity of this statement is presented in Appendix I. The energy quantity $U_{m, 4}$ for the preceding example can be worked out via Equation (12b) or as the total area under the force-displacement (push-over) curve of Figure 4, i.e., $U_{m, 4}=6.9512\left[\left(M^{P}\right)^{2} L / E I\right]$. Equation (12d) can then be used to compute the total internal energy of the entire system as; $U_{4}=(8 F h / F h) U_{m}=55.6096\left[\left(M^{P}\right)^{2} L / E I\right]$, or in terms of ultimate values; $\phi_{4}=\phi^{P}$ and $F_{4}=F^{P}$ at incipient collapse as; $U_{4}=(55.6096 / 0.7386 \times 16) F^{P} h \phi^{P}=4.7057 F^{P} h \phi^{P} . U_{4}$ can also be looked upon as an indication of the capacity of the structure to absorb external energy.

\subsubsection{Stiffness Degradation}

An understanding of the rate and sequence of degradation of story level stiffnesses $\partial_{s} K_{i, s}$, is a priori to estimating the momentary periods, $\partial_{s} T_{s}$, of vibrations of the system at any response stage " $s$ ". Progressive plasticity tends to degrade the global stiffness and modify the dynamic characteristics of statically indeterminate structures under monotonically increasing lateral forces. The effects of stiffness degradation are more pronounced in MFUR since many members of similar characteristics either, fail, become inactive or develop plastic hinges simultaneously. The natural period of vibration of each stage of global loss of stiffness increases with advancing stages of loading until the structure ceases to resist external forces. As the rate of degradation of global stiffness is a function of increasing number of plastic hinges, Equations (5d) and (5e) may be rearranged to assess the gradual loss of global stiffness in terms of sequential formation of plastic hinges.

To demonstrate the use of Equations (5d) and (5e), consider the long hand stiffness analysis of the 3rd story of the MFUR of example I for all four stages of response. The results of this rather cumbersome exercise for $f_{C R . m, s}=1$ may be summarized as; $K_{3,1}=27.70 K_{0}, K_{3,2}=20.17 K_{0}$, $K_{3,3}=12.31 K_{0}$ and $K_{3,4}=7.80 K_{0}$, where $K_{0}=E I / L h^{2}$. However, since in MFUR the distribution of story level stiffness is also a function of the story level shears, it would be reasonable to seek a simpler method of computing for the story level stiffnesses in terms of shear force ratios at different stages of loading, i.e.,

$$
\left(\partial_{s} K_{i, s} / K_{m, s}\right)=\left(\partial_{s} V_{s, i} / \partial_{s} V_{m, s}\right) \times\left(h_{m} / h_{i}\right)
$$

A derivation of Equation (13a) is presented in the Appendix II. This Equation implies that:

The ratio of stiffness of any two floors of an MFUR is proportional to the ratio of shear forces of the two levels multiplied by the inverse ratio of their heights.

The use of Equation (13) can be demonstrated by the following simple computations:

$$
K_{1,1}=(3 F / F)(h / 1.5 h) K_{m, 1}=55.40 K_{0}, K_{2,1}=(2 F / F)(h / 1.25 h) K_{m}=44.32 K_{0}, \text { etc. }
$$

To further illustrate the applications of the proposed solutions consider the deterioration of the story level stiffnesses of the example frame at all four stages of loading. Since changes in the global drift angle are influenced directly by degradation of story level stiffnesses, then the story level stiffness of 
any response stage can be associated with the drift angle of the same stage. Therefore, the results of group of Equations (11b) can be used to compute all $K_{i, s}$ by simple, numerical proportioning e.g.,

$$
\begin{array}{llll}
K_{3,1}=27.70 K_{0} & K_{3,2}=20.17 K_{0} & K_{3,3}=12.31 K_{0} & K_{3,4}=7.80 K_{0} \\
K_{2,1}=44.32 K_{0} & K_{2,2}=32.27 K_{0} & K_{2,3}=19.70 K_{0} & K_{2,4}=12.48 K_{0} \\
K_{1,1}=55.40 K_{0} & K_{1,2}=40.34 K_{0} & K_{1,3}=24.62 K_{0} & K_{1,4}=15.60 K_{0}
\end{array}
$$

This set of numbers complete the stiffness degradation matrix of the subject example frame.

\subsubsection{Period Analysis}

The dynamically induced seismic forces of MFUR are highly sensitive to variations in the fundamental period of vibrations as well as the shape and magnitude of the pre-assigned drift profile. Both of these issues are briefly discussed in this section. The period analysis presented herein is based on the following basic assumptions:

1. The normalized displacement function $\psi_{i, s}=\bar{h}_{i} / H$ remains unchanged throughout the loading history of the structure. In other words, loss of stiffness changes only the magnitude of lateral displacements, but not the deformed shape $\partial_{s} \phi_{s} \times \bar{h}_{i}$ of the system.

2. The lateral displacement profile of the frame is a function of the single variable $\phi_{s}$ for all stages of loading, i.e., all displacement profiles $\partial_{s} \Delta_{i, s}=\partial_{s} \phi_{s} \cdot \bar{h}_{i}$ follow the same linear shape function as their normalized displacement function $\psi_{i, s}=\psi_{s}=\bar{h}_{i} / H$.

3. Dominant mode shapes remain unchanged during formation of plastic hinges and that the coupling of modes in the inelastic range can be neglected.

4. The first mode of the first un-degraded stage is the most dominant mode of all response stages and that the first mode of each stage is the dominant mode of that stage.

The fundamental period of vibrations of any stable MFUR corresponding to any particular response stage can be expressed as: $\partial_{s} T_{s}=T_{s}=2 \pi \sqrt{\bar{M}_{s}^{*} / \partial_{s} K_{s}{ }^{*}}$, where, the generalized stiffness and mass of the $s^{\text {th }}$ response stage are defined as:

$$
\partial_{s} K_{s}^{*}=\sum_{i=1}^{m} \partial_{s} K_{i, s} \times\left(\frac{\partial}{\partial \bar{h}_{i}} \psi_{i}\right)^{2} \text { and } M^{*}=\sum_{i=1}^{m} \bar{M}_{i} \times \psi_{i}^{2} \text { respectively }
$$

Assuming $\quad \bar{M}_{i}=\bar{M}=W / g \quad$ and $\quad$ substituting $\quad \sum_{i=1}^{m} \partial_{s} K_{i, s}=\left(\partial_{s} K_{m, s} h_{m} / \partial_{s} V_{m, s}\right) \sum_{i=1}^{m}\left(\partial_{s} V_{i, s} / h_{i}\right) \quad$ and $\left(\frac{\partial}{\partial \bar{h}_{i}} \psi_{i}\right)^{2}=\left(\bar{h}_{i}-\bar{h}_{i-1} / H\right)^{2}=\left(h_{i} / H\right)^{2}$ in Equation (14a) gives;

$$
T_{s}=2 \pi\left\{\left[\sum_{i=1}^{m} \bar{M}_{i} \times \bar{h}_{i}^{2}\right] /\left(\left(\frac{\partial_{s} K_{m, s}}{\partial_{s} V_{m, s}}\right) \sum_{i=1}^{m}\left(\frac{\partial_{s} V_{i, s}}{h_{i}}\right)\left(\frac{h_{i}}{H}\right)^{2}\right]\right\}^{1 / 2}
$$

Observing that the normalized displacement function is independent of sequence of formation of plastic hinges, and that the term $M^{*}=\sum_{i=1}^{m} \bar{M} \times \bar{h}_{i}^{2}$ and the ratio $\left(h_{m} / \partial_{s} V_{m, s}\right) \sum_{i=1}^{m}\left(\partial_{s} V_{i, s} / h_{i}\right)$ are constant for all " $s$ ", then $T_{s}$ can be expressed in terms of the single variable, $K_{m, s}$, i.e., 


$$
T_{s}=C \sqrt{\bar{M} / K_{m, s}}
$$

Where, $C$ is a numerical constant. Equation (14c) indicates that MFUR can be treated as single degree of freedom (SDOF) systems for all "s", and all practical purposes. Since both $C$ and $\bar{M}$ are independent of " $s$ ", once the fundamental period of vibrations of the un-degraded structure, $T_{1}$, is determined the corresponding values for each degraded stage can be worked out through simple proportioning,

$$
T_{s}=T_{1} \sqrt{\left(K_{m, 1} / K_{m, s}\right)}
$$

To demonstrate the applications of Equations (14b) and (14d), consider the variations of fundamental period of vibrations of the example frame with respect to changes in the global stiffness of the structure. Equation (14b) gives:

$$
T_{1}=2 \pi\left\{\left[\frac{\left(1.5^{2}+2.75^{2}+3.75^{2}\right) \bar{M}}{3.75^{2}}\right] /\left[\left(\frac{27.07 K_{0}}{12.1524}\right)\left(\frac{1 \times 1+2 \times 1.25+3 \times 1.5)}{3.75^{2}}\right) 12.1524\right]\right\}^{\frac{1}{2}} \text {, or } \quad T_{1}=2.06 \sqrt{\bar{M} / K_{0}} \text {. }
$$

Equation (14d) can now be used to determine $T_{2}, T_{3}$ and $T_{4}$ as:

$$
T_{2}=T_{1} \sqrt{27.70 / 20.17}=1.17 T_{1}, T_{3}=T_{1} \sqrt{27.70 / 12.31}=1.50 T_{1}
$$

and

$$
T_{4}=T_{1} \sqrt{27.70 / 7.80}=1.89 T_{1} .
$$

The elongation of the natural period of vibrations is associated with the loss of global stiffness. Degradation of the stiffness reduces the rate of change of demand as well as the reserve capacity of the remaining (intact) structure. As the reserve capacity and the rate of change of demand diminish, the structure becomes softer until it fails through a collapse mechanism.

\subsubsection{Energy Equivalency}

Since by virtue of $\left(\Delta_{m} / \delta_{m}=H / h_{m}\right)$, the global stiffness of the structure is in direct proportion with each story level stiffness, and that by definition, Equation (1a), the lateral displacements are a function of the single variable $\phi_{s}$ for all " $s$ " then MFUR may be looked upon as SDOF systems for all practical purposes.

In short; MFUR may be treated as statically determinate, SDOF structures.

This implies that Equation (12d) can be used in conjunction with Housner's [9] equal energy concept for SDOF structures in order to formulate the demand-capacity relationship of the subject frame in terms of its seismic shears and the corresponding internal energy generated within the structure at any response stage "s", i.e.,

$$
\frac{\gamma}{2}\left(\frac{Q}{g}\right)\left(\frac{T_{s}}{2 \pi} S_{a} g\right)^{2}=U_{s}=\left(U_{m, s} / M_{m, s}^{R}\right) M_{O, s}
$$


$S_{a}$ and $\gamma=\left(2 \mu_{S}-1\right) / R_{\mu}^{2}$ are the spectral acceleration and the energy equivalency factors respectively [10]. $\mu_{S}=\Delta_{m, s} / \Delta_{m, s=1}$ and $R_{\mu}$ are defined as the period dependant ductility and ductility reduction factors respectively. If the quantity $U_{s}$ is interpreted as the seismic capacity of the structure, then the right hand side of Equation (15), may be looked upon as the seismic demand or equivalent total dynamic input energy of the system.

\subsection{Demonstrative Example III}

Compute the base shear of the moment frame of example I subjected to seismically induced lateral forces of uniform intensity $F$ at first yield, $s=1$, and at incipient collapse, $s=4$, in terms of the following design data;

Prescribed design drift ratios $\phi_{Y} \leq 0.01$ and $\phi^{p} \leq 0.02$ radians, total structural self weigh $Q=3 \mathrm{~W}$ and un- medium degraded range fundamental period of vibrations, $T_{s=1}=S_{D 1} / S_{a}$, where, $S_{D 1}$ is the site specific design spectral response acceleration parameter. Substituting for the corresponding quantities and the total internal energies $U_{1}=U_{Y}=4.00 F_{Y} h \phi_{Y}$ at first yield and $U_{4}=U^{P}=4.7057 F^{P} h \phi^{P}$ at incipient collapse in Equation (15), and bearing in mind that $V=3 F$, it gives:

$$
V_{Y}=\left(\frac{\gamma g S_{D 1}^{2}}{0.01 h 4 \pi^{2}}\right) \frac{9 W}{8.00}=2.85 \gamma g S_{D 1}^{2} W
$$

and

$$
V^{P}=\left(\frac{\gamma g S_{D 1}^{2}}{0.02 h 4 \pi^{2}}\right)^{2} \frac{9 W}{9.415}=1.21 \gamma g S_{D 1}^{2} W
$$

respectively.

\section{Conclusions}

It was shown that SUR in general and MFUR in particular are ideally suited for Performance Based Elastic-Plastic Design. A number of new, closed form formulae for understanding the response of MFUR were presented. The proposed methodology lends itself well to controlling the sequential response of MFUR due to monotonically increasing lateral forces. MFUR approach results in Unique Minimum Weight solutions for lateral force resisting moment frames designed to perform as intended at any prescribed response stage. Because of their predetermined characteristics, MFUR can be treated as statically determinate, SDOF structures ideally suited for energy equivalency analysis. It was demonstrated through simple parametric examples that the proposed procedures provide useful design information that neither elastic nor plastic methods of analysis can offer on their own. Furthermore, it was shown that the sequences of formations of plastic hinges could be controlled by selecting the relative stiffness of groups of similar beams in accordance with predetermined performance objectives. The more significant theoretical aspects of MFUR were summarized in the following statements:

- In MFUR selected groups of beams and columns share the same drift and Demand-capacity ratios. 
- The ultimate load carrying capacity of an $(m \times n) M F U R$ with moment resisting grade beams under lateral loading of apex value $F^{P}$ is $M^{P}=F^{P} h / 4 n$.

- The ratio of total internal energy of MFUR to that of anyone of its levels, such as the roof, is equal to the ratio of the global overturning moment to the overturning moment of that (roof) level.

- The ratio of stiffness of any two floors of an MFUR is proportional to the ratio of shear forces of the two levels multiplied by the inverse ratio of their heights.

- MFUR may be treated as statically determinate, SDOF structures.

Most importantly, the proposed formulations may help engineers gain insight into structural behavior of lateral load resisting SUR and lend themselves well to manual as well as spreadsheet computations.

In closing it should be emphasized that the presented approach may be used for the preliminary economical performance based design of lateral force resisting moment frames. The final design may need to be checked and verified using an appropriate inelastic dynamic or static analysis.

\section{References}

1. Goel, S.C.; Liao, W.; Bayat, M.R.; Chao, S. Performance-based plastic design method for earthquake resistant structures. Struct. Des. Tall Spec. Build. 2010, 19, 115-137.

2. Mazzolani, F.; Pilosu, V. Theory and Design of Seismic Resisting Moment Frames; Taylor \& Francis: Oxford, UK, 1996.

3. Grigorian, M.; Grigorian, C. Performance control for seismic design of moment frames. J. Construct. Steel Res. 2011, 67, 1106-1114.

4. Grigorian, M.; Grigorian, C. Recent developments in plastic design analysis of steel moment frames. J. Construct. Steel Res. 2012, in press.

5. Grigorian, M. On the lateral response of regular high-rise frames. Struct. Des. Tall Spec. Build. 1993, 2, 233-252.

6. Grigorian, M.; Grigorian, C. Preliminary minimum weight design of moment frames under lateral loading. AISC Eng. J. 1988, 25, 129-136.

7. Beedle, L.S. Plastic Design of Steel Frames; John Wiley \& Sons: Hoboken, NJ, USA, 1985.

8. Neal, B.G. The Plastic Methods of Structural Analysis; Chapman \& Hall Ltd.: London, UK, 1963.

9. Housner, G.W. Limit Design of Structures to Resist Earthquakes. In Proceedings of the 1st World Conference on Earthquake Engineering, Berkeley, CA, USA, 1956; Earthquake Engineering Research Institute (EERI): Berkeley, CA, USA, 1956.

10. Chopra, A.K.; Goel, R.K. Capacity-demand diagram methods for estimating seismic deformations of inelastic structures. Earthq. Spectra 1999, 15, 637-655.

\section{Appendix I}

In order to verify the validity of the total internal energy expression, $U=\left(U_{m} / M_{m}^{R}\right) M_{O}$, consider the ratio of internal energy of any level " $i$ " to that of the roof level " $m$ ", i.e.,

$$
\left(U_{i} / U_{m}\right)=\left(V_{i} / V_{m}\right)^{2}\left(K_{m} / K_{i}\right)
$$


However, since the drift ratio is constant, i.e., $\phi_{i}=\phi_{m}$, then $\left(K_{m} / K_{i}\right)=\left(V_{m} / V_{i}\right)\left(h_{i} / h_{m}\right)$. Substituting for $\left(K_{m} / K_{i}\right)$ in Equation (16a), it gives $\left(U_{i} / U_{m}\right)=\left(V_{i} h_{i} / F_{m} h_{m}\right)=\left(M_{i}^{R} / M_{m}^{R}\right)$. The total internal energy of the system may also be computed as the sum of internal energies of the " $m$ " individual levels, i.e.,

$$
U=\sum_{i=1}^{m} U_{i}=\left(U_{m} / M_{m}^{R}\right) \sum_{i=1}^{m} M_{i}^{R}=\left(U_{m} / M_{m}^{R}\right) M_{O}
$$

\section{Appendix II}

The local Force- displacement relationship of any story level " $i$ " can be expressed in terms of its local drift angle as $\delta_{i, s}=\phi_{i, s} h_{i}=V_{i, s} / K_{i, s}$, and for the roof level as $\delta_{m, s}=\phi_{m, s} h_{i}=V_{m, s} / K_{m, s}$. Observing that by definition, $\phi_{i, s}=\phi_{m, s}$, then the division $\left(\delta_{i, s} / \delta_{m, s}\right)$ gives; $\left(K_{i} / K_{m}\right)=\left(V_{i} \times h_{m} / V_{m} \times h_{i}\right)$.

(C) 2012 by the authors; licensee MDPI, Basel, Switzerland. This article is an open access article distributed under the terms and conditions of the Creative Commons Attribution license (http://creativecommons.org/licenses/by/3.0/). 